\title{
Médiévales
}

Langues, Textes, Histoire

49 | automne 2005

La paroisse, genèse d'une forme territoriale

\section{La délimitation des territoires paroissiaux dans les pays de moyenne Garonne ( $\mathrm{e}^{\mathrm{e}}-\mathrm{XV}^{\mathrm{e}}$ siècles)}

The process of bounding parish territories in Quercy (10th-15th c.).

\section{Florent Hautefeuille}

\section{(2) OpenEdition}

Journals

Édition électronique

URL : https://journals.openedition.org/medievales/1290

DOI : 10.4000/medievales. 1290

ISSN : 1777-5892

Éditeur

Presses universitaires de Vincennes

Édition imprimée

Date de publication : 1 décembre 2005

Pagination : 73-88

ISBN : 2-84292-177-1

ISSN : 0751-2708

Référence électronique

Florent Hautefeuille, "La délimitation des territoires paroissiaux dans les pays de moyenne Garonne ( $\mathrm{x}^{\mathrm{e}-\mathrm{xv}}$ e siècles) », Médiévales [En ligne], 49 | automne 2005, mis en ligne le 05 mars 2008, consulté le 22 avril 2022. URL : http://journals.openedition.org/medievales/1290 ; DOI : https://doi.org/10.4000/ medievales. 1290

Ce document a été généré automatiquement le 22 avril 2022

Tous droits réservés 


\title{
La délimitation des territoires paroissiaux dans les pays de moyenne Garonne ( $\mathrm{x}^{\mathrm{e}}-\mathrm{XV}^{\mathrm{e}}$ siècles)
}

The process of bounding parish territories in Quercy (10th-15th c.).

\author{
Florent Hautefeuille
}

1 L'histoire de la paroisse telle qu'elle fut initiée par P. Imbart de la Tour ${ }^{1}$ rattache cette entité aux origines du christianisme, ou plutôt aux origines du christianisme officiel (IV siècle). C'est le décalage entre cette paroisse presque «immémoriale » et la réalité des actes de la pratique qui avait été un des moteurs de ma thèse de doctorat sur le peuplement rural et la mise en place du réseau paroissial en bas Quercy, soutenue en $1999^{2}$. La paroisse semblait avoir eu une existence théorique dans les textes normatifs et la législation ecclésiastique, mais était pratiquement absente des corpus de chartes avant l'extrême fin de l'époque carolingienne.

2 Cet article reprend une partie des conclusions de ma thèse, tout en cherchant à préciser certains points éclairés par un renouvellement important des recherches sur ce thème depuis quelques années ${ }^{3}$. Je concentrerai mon propos sur une aire géographique restreinte, soit le bassin de la moyenne Garonne, entre les rivières du Lot, du Tarn et de l'Aveyron. Ce secteur présente l'avantage d'offrir un paysage varié tant d'un point de vue géographique que politique: des vallées assez fertiles séparées par des serres plus arides (pays de Vaux au nord de l'Aveyron); une plaine alluviale ayant connu une anthropisation précoce et intense (vallées du Tarn, de l'Aveyron et de la Garonne); des coteaux intermédiaires couverts de forêts jusqu'à un Moyen Âge avancé (forêt d'Agre et de Tulmon ${ }^{4}$ ).

3 Historiquement, cette aire correspond au nord de la zone d'influence des comtes de Toulouse. C'est à cette époque qu'est fondée la ville de Montauban, une des plus belles réussites urbaines du XII ${ }^{\mathrm{e}}$ siècle, aux marges des diocèses de Cahors et de Toulouse, dans une zone qui connait un essor économique très vif $d u$ XII ${ }^{e}$ au début du XIV ${ }^{\mathrm{e}}$ siècle. La présence des abbayes Saint-Théodard-de-Montauriol et de Moissac, puis de l'évêché de 
Montauban à partir de 1317, est à l'origine d'une riche documentation aussi bien sous la forme d'un cartulaire à Saint-Théodard ${ }^{5}$ que d'originaux à Moissac ${ }^{6}$. Mon analyse repose également sur le dépouillement de quelques chartriers laïques tel que celui de la châtellenie de Castelnau-Montratier dans le Lot. La masse documentaire est parfois étonnante. Certaines petites paroisses de moins de 1000 hectares sont couvertes par plusieurs centaines de textes médiévaux essentiellement après 1250. C'est le cas de la paroisse de Flaugnac sur laquelle, parallèlement au dépouillement des sources écrites, je mène des recherches archéologiques dans le cadre d'un projet collectif de recherche. Cela permet une approche spatiale fine et compense partiellement l'absence de cartographie même moderne des limites paroissiales ${ }^{7}$.

4 En m'appuyant sur cette riche documentation, j'essaierai de préciser les contours tant physiques que théoriques de la paroisse de la seconde moitié du Moyen Âge. À partir de ces éléments de définition, je reviendrai sur les différentes hypothèses que l'on peut avancer pour expliquer le processus de mise en place, puis de stabilisation, du maillage des paroisses.

Le statut des paroisses rurales aux XIII ${ }^{\mathrm{e}}$-XV ${ }^{\mathrm{e}}$ siècles

5 Le processus de définition de la paroisse rurale est complexe et long ${ }^{8}$. On peut cependant considérer que les définitions qui apparaissent au XIII ${ }^{\mathrm{e}}$ siècle ${ }^{9}$ forgent un outil théorique désormais abouti et surtout à peu près appliqué. Il convient pourtant de s'attarder sur le statut des très nombreuses églises qui apparaissent dans la documentation de la fin du Moyen Âge. Il existe pour le Quercy un pouillé de l'extrême fin du XIII ${ }^{\mathrm{e}}$ siècle ou des premières années du siècle suivant ${ }^{10}$ qui permet d'avoir une vision globale du diocèse. Même s'il s'agit d'un document fiscal, le vocabulaire utilisé pour désigner les bénéfices est d'une assez grande variété. Tous ces bénéfices ne sont pourtant pas assimilables à une entité paroissiale. Au total, et sans distinction de statut, on peut dénombrer exactement 900 bénéfices pour l'ensemble du diocèse de Cahors. Il semble que le terme servant à désigner un bénéfice assimilable à une église paroissiale simple est celui d'ecclesia. Ce terme est utilisé 502 fois. On trouve également, mais de façon plus exceptionnelle, des ecclesiae parochiales ${ }^{11}$ et deux cas de parochia ${ }^{12}$. Le rapprochement avec les actes de la pratique permet de vérifier que ces bénéfices correspondent à des entités paroissiales dotées d'une église, d'un cimetière et d'un territoire défini sur lequel réside la communauté. Elles ont à leur tête un curé.

6 À côté de cette catégorie, le pouillé fait aussi apparaître un très grand nombre de capellae, capellaniae ou capellani. Contrairement à l'acception usuelle actuelle, ces chapelles ne correspondent pas, ou très rarement, à des édifices secondaires dépourvues de la cura animarum. Ce ne sont pas non plus des églises soumises à une ecclesia matrix. Ainsi, la capellania Montis Dominici ${ }^{13}$ correspond à l'église paroissiale de Montdoumerc ${ }^{14}$. Cette église entre dans la documentation écrite à la fin $\mathrm{du} \mathrm{XI}^{\mathrm{e}}$ siècle à l'occasion d'un conflit violent entre l'abbé de Saint-Théodard et l'évêque de Cahors ${ }^{15}$. Les dîmes sont déjà mentionnées et sont un des éléments du conflit. Par ailleurs, l'édifice est à l'origine de la formation d'un des villages ecclésiaux les plus aboutis de la région. Le bourg est doté de remparts avant le milieu du XIII ${ }^{\mathrm{e}}$ siècle ${ }^{16}$. La paroisse s'étend sur plus de 800 hectares. Aucun document n'évoque l'hypothèse d'un lien de dépendance de cette église vis-à-vis d'une église voisine. En réalité, tout laisse penser que ces capellae correspondent autant à des églises paroissiales que les ecclesiae. La plupart d'entre elles peuvent être associées à un territoire délimité, à un cimetière, à un décimaire autonome. 
$7 \quad$ L'origine de ces distinctions nominales ne semble pas liée au statut paroissial ou non de l'église, mais plutôt à la présence d'un prieuré-cure. Ce type de structure ecclésiale est fréquent en Quercy mais semble se traduire dans la plupart des cas par une simple division des revenus de la cure entre un rector et un prior. Les deux charges sont d'ailleurs parfois regroupées sur les épaules d'un seul individu. Dans le pouillé, l'association entre capella et prior n'est pas systématique mais très fréquente. En outre, plusieurs indices montrent que cette association n'est pas le fait du hasard. La capella n'est autre que la partie du bénéfice qui n'est pas concernée par les revenus du prieur. Ainsi, dans le cas de l'église de Calviac, dans le nord du Quercy, il est précisé que le bénéfice de la chapelle n'a aucune valeur car le prieur a tout pris ${ }^{17}$. C'est cette situation qui explique également le décalage fiscal existant entre les ecclesiae, les priores et les capellaniae. $67 \%$ des prieurs sont exempts de la décime alors que seules 1,6\% des ecclesiae le sont. De même, alors que $20 \%$ des ecclesiae sont dites indécimables, on s'aperçoit que $70 \%$ des capellae le sont. Cela ne signifie nullement que ces paroisses sont moins peuplées, voire désertées, mais seulement que le prieur prélève une part importante des revenus.

8 Le pouillé ne permet pas de distinguer clairement des églises paroissiales et des églises non paroissiales. Il faut donc s'appuyer également sur les actes de la pratique pour classer en trois catégories principales cette masse d'églises.

9 1. L'ecclesia parochialis de base correspond en bas Quercy à une église généralement isolée, parfois proche d'un mas, plus rarement au centre d'un village. Son territoire est progressivement défini entre le $\mathrm{XI}^{\mathrm{e}}$ et le $\mathrm{XIII}^{\mathrm{e}}$ siècle. Elle est associée à un décimaire correspondant à son emprise spatiale. Ses contours précis peuvent parfois se traduire par un territoire discontinu avec des séries d'enclaves qu'il n'est pas toujours aisé de repérer. Au XIII ${ }^{\mathrm{e}}$ siècle, elle est théoriquement dotée d'un prêtre nommé rector, presbyter ou gubernator. Ce type de paroisse est numériquement dominant sur l'ensemble du bas Quercy mais aussi dans une bonne partie de l'Agenais et du Rouergue. De ce fait, le maillage paroissial médiéval et moderne est beaucoup plus resserré que celui des communes actuelles. En moyenne, les communes contemporaines recouvrent de 4 à 5 paroisses médiévales. Dans certaines grandes communes, on peut même observer jusqu'à 15 ou 20 paroisses ${ }^{18}$.

10 2. La seconde catégorie d'églises paroissiales est celle des prieurés-cures. Il s'agit globalement d'édifices et de paroisses qui physiquement et topographiquement ne devaient pas se distinguer de celles de la première catégorie. Il est impossible de différencier un prieuré-cure d'une simple église paroissiale par l'étendue de son territoire, son ancienneté ou l'importance des bâtiments cultuels. Le prieuré-cure n'a pas non plus de lien particulier avec un établissement monastique. Nombre d'entre eux sont placés sous sous le patronage de l'évêque. Le seul élément de distinction est donc la division en deux parts de ses revenus. Une part du bénéfice revenait au prieur, une autre part, assimilable à la portion congrue, était réservée à la cure. Très souvent il semble que le curé soit également le prieur. Parfois, on voit cependant apparaitre un vicaire chargé du culte. Dans ces cas-là le prieur ne réside pas, du moins à partir du XIV siècle. Ce système a été largement mis à profit par les papes français, en particulier par Jean XXII, pour couvrir de bénéfices sa parentèle élargie. Dans ses travaux sur les familles quercynoises du XIV $v^{\mathrm{e}}$ siècle, le chanoine Albe a mis en évidence ce phénomène. Les cadets de familles disposaient ainsi fréquemment des revenus de prieurés, situés parfois sur les terres familiales, mais aussi parfois très éloignés de ces dernières ${ }^{19}$. 
11 3. À ces deux catégories principales, il faut ajouter une partie au moins des prieurés réguliers dépendant d'établissements monastiques tels que Moissac, Saint-Théodard, Marcilhac ou Saint-Sernin-de-Toulouse. Beaucoup moins nombreux, ils se distinguent par la présence d'une petite communauté religieuse, parfois très réduite. Il arrive que cette dernière disparaisse précocement, ce qui complique encore les identifications ${ }^{20}$. Pour ces prieurés réguliers, se pose le problème de l'unicité de l'édifice cultuel. Des indices assez nombreux permettent d'envisager pour certaines d'entre eux une église paroissiale distincte de l'église prieurale ${ }^{21}$. Là encore, bien souvent la disparition de la communauté monastique entraîne celle de l'un des deux édifices. Mais en dehors d'une riche documentation ou d'une enquête archéologique fine, il est souvent difficile d'identifier clairement ces cas ${ }^{22}$.

Ce phénomène des églises doubles générant des paroisses bicéphales est observable ailleurs, parfois sans le support d'un prieuré. C'est en particulier le cas des nombreux villages castraux à partir des $\mathrm{XII}^{\mathrm{e}}$-XIII ${ }^{\mathrm{e}}$ siècles. Ces villages, mais c'est aussi le cas d'une majorité de bastides au $\mathrm{xIII}^{\mathrm{e}}$ siècle, s'installent sur des territoires où le maillage des paroisses est déjà en place. De ce fait, ces villages ne sont pas dotés d'une église paroissiale. Certains ont d'ailleurs traversé le Moyen Âge, voire l'époque moderne, sans église. La population continue à se rendre à l'église champêtre parfois située à plusieurs dizaines de minutes de marche. C'est par exemple le cas d'un village comme Belfort en Quercy ${ }^{23}$, pourtant chef-lieu de châtellenie. Mais il arrive aussi, selon un processus dont le détail nous échappe souvent, que se produise un remodelage partiel du maillage paroissial de certains de ces castra ${ }^{24}$. Ce processus du glissement du centre paroissial est connu par de nombreux exemples ${ }^{25}$. Parfois, il se traduit par une demande formelle de transfert du centre paroissial ancien vers la chapelle intra-muros. Dans ces cas-là, on peut penser que le processus est plus rapide. Les registres pontificaux nous livrent d'assez nombreuses demandes allant dans ce sens à partir de la fin du XIII ${ }^{e}$ siècle et, surtout, au XIV ${ }^{e}$ siècle. Le transfert du centre paroissial ne paraît pas avoir eu d'impact sur le territoire paroissial. C'est par exemple le cas du castrum de Durfort, situé dans l'orbite de l'abbaye de Moissac. Cette autorisation de transfert de siège est demandée et obtenue en $1311^{26}$.

Hiérarchie des centres paroissiaux?

13 Au-delà du problème des différents types d'églises rencontrés dans la documentation écrite, il me semble important de s'arrêter également sur le phénomène très répandu, à la fin du Moyen Âge, des églises mères et des églises annexes. On a parfois tendance à rattacher ce phénomène à une situation héritée du haut Moyen Âge et à faire des églises mères les héritières des paroisses primitives ${ }^{27}$. Cette filiation semble peu vraisemblable. Les relations de matrices à annexes sont rares dans la documentation des $\mathrm{XI}^{\mathrm{e}}$ et $\mathrm{XII}^{\mathrm{e}}$ siècles ${ }^{28}$. Les mentions ne se multiplient qu'à partir de la fin du XII et au $\mathrm{XIII}^{\mathrm{e}}$ siècle. Il n'est alors pas rare de voir une église en dominer une, deux, voire trois ou quatre autres. Ce phénomène est très répandu dans les pays d'habitat dispersé, où la polarisation de l'habitat est incomplète ou nulle. Les paroisses concernées sont souvent de petites dimensions. En bas Quercy, ce schéma est fréquent, mais ne renvoie nullement à des entités paroissiales aux prérogatives incomplètes. En effet, la hiérarchie existe bien mais ne recouvre concrètement que le desservant, sans aucun impact sur la cohérence et l'autonomie des paroisses les unes par rapport aux autres. Dans les centaines de contrats agraires que j'ai pu dépouiller pour cette zone, jamais on ne distingue une église paroissiale matrice d'une simple annexe. Les territoires 
paroissiaux des églises annexes restent parfaitement délimités, et chaque église conserve sa communauté, son cimetière et son décimaire autonome. Elle est même dotée d'un prêtre souvent distinct du desservant de sa matrice. Mais ce prêtre n'a alors que le titre de vicaire. L'église mère est l'église où réside le rector en titre. Si ce dernier décide de s'installer dans l'une de ses annexes, cette dernière devient alors église mère et l'ancienne église mère est rétrogradée au rang de simple annexe. Cela relativise grandement l'importance de ces hiérarchies.

documentaire assez bien fourni permet d'illustrer ce processus à travers un exemple situé à une dizaine de kilomètres à l'est de la ville de Montauban. En 1284, l'abbé de Saint-Théodard donne au prieur de Courondes les dîmes non pas de cette paroisse, mais de la paroisse voisine de Castanèdes ${ }^{29}$. On peut penser que cette donation est à l'origine de la situation attestée autour de 1300, où Castanèdes apparaît comme une annexe de Courondes. Mais l'union de ces deux paroisses voisines ne semble concerner que les cures et pas les prieurés. Le pouillé de 1300 fait ainsi apparaître un prieur distinct pour les deux entités. Soixante-dix ans plus tard, ce même prieuré de Castanèdes se trouve désormais uni, non pas à l'église de Courondes, mais à une autre église paroissiale voisine, celle de Nègrepelisse, bastide fondée à la fin du XIII siècle. Ce lien semble avoir été très éphémère. Il n'apparait plus après la guerre de Cent Ans. En revanche, la sujétion de Castanèdes vis-à-vis de Courondes s'est inversée et cette dernière est signalée comme l'annexe de Castanèdes. Ces exemples sont fréquents en Quercy ${ }^{30}$ durant les trois derniers siècles du Moyen Âge. Ces rapprochements fragiles et provisoires paraissent avoir été justifiés par des raisons économiques. Il s'agit de regroupements de bénéfices destinés à asseoir des revenus plus élevés aux curés en titre.

De ce fait, la hiérarchie des églises dans le système paroissial ne paraît pas avoir eu d'impact sur le mode de fonctionnement concret des paroisses. Si d'autres lieux de culte apparaissent assez fréquemment à partir du XIV siècle (chapelles isolées ou liées à des hôpitaux ou des léproseries), il n'est jamais difficile de les distinguer des églises paroissiales. En revanche, apparaissent assez fréquemment dans la documentation écrite, et ce dès le $\mathrm{xI}^{\mathrm{e}}$ siècle, des mentions de dîmes et de décimaires qui ne semblent pas toujours liés à des paroisses. Encore en 1300, le pouillé signale quelques bénéfices qui se limitent à un décimaire. C'est le cas de celui de Sesquières près de Caussade ${ }^{31}$. Cet espace correspond initialement à un ensemble de terre dépendant d'une grange templière de la commanderie de Vaours. Ce décimaire a fini par se transformer très tardivement en entité paroissiale ${ }^{32}$. Mais nous ignorons à quelle paroisse était rattaché ce petit territoire au Moyen Âge. D'autres exemples de décimaires autonomes n'ont pas connu cette évolution et n'ont laissé aucune trace dans l'organisation paroissiale tardomédiévale et moderne. Certains sont mentionnés dès le $\mathrm{xl}^{\mathrm{e}}$ siècle; d'autres ont $\mathrm{pu}$ continuer à vivre jusqu'à l'époque moderne, généralement parce que ces dîmes avaient un destinataire distinct du curé de la paroisse. C'est le cas d'un petit décimaire sur la commune de Bressols, pour lequel nous disposons de plans faits au XVIII ${ }^{e}$ siècle. Il ne fait aucun doute que ces décimaires n'ont jamais été liés ni à une église ni à un cimetière. Certains, en revanche, ont pu correspondre à ces minuscules communautés que constituaient les mas. Il s'agit assez vraisemblablement des séquelles des trafics de dîmes qui apparaissent régulièrement dans les cartulaires aux $\mathrm{XI}^{\mathrm{e}}$ et $\mathrm{XII}^{\mathrm{e}}$ siècles. Cette particularité des décimaires de mas a pu cependant avoir un impact sur la genèse territoriale de certaines paroisses. 
Les processus de territorialisation consécration d'églises paroissiales ${ }^{33}$. Les mentions de délimitation de paroisses apparaissent donc au détour de conflits plus que comme un élément constitutif d'un système. Le mot parochia est lui-même absent de la documentation carolingienne. Sa première apparition en bas-Quercy ne date que du règne de Henri I ${ }^{\text {er }}$. Cette apparition tardive dans les actes de la pratique d'une réalité que les textes normatifs font apparaître plus tôt, n'est pas propre au Quercy. À Lézat, le terme n'apparaît de façon certaine qu'à la fin du xe siècle ${ }^{34}$, mais il reste exceptionnel avant les années 1030-1050. À cette date, en revanche, on observe une véritable explosion du nombre d'occurrences. Pendant le règne d'Henri I ${ }^{\text {er }}, 14,9 \%$ des textes mentionnent au moins une parochia. En outre, P. Ourliac et A.-M. Magnou remarquent l'absence totale $\mathrm{d}$ 'allusions à des limites de décimaire au $\mathrm{x}^{\mathrm{e}}$ siècle dans le cartulaire de Lézat ${ }^{35}$. Il serait sans doute nécessaire de mener une enquête à grande échelle sur la chronologie d'apparition des dîmes dans les actes de la pratique. Des sondages réalisés dans les cartulaires des régions voisines (Toulousain, Limousin, Rouergue) ${ }^{36}$ font apparaître une chronologie d'apparition assez proche de celle de la parochia.

fut egalement constater que, si l'apparition de ces termes semble traduire une évolution sensible dans la structuration des entités paroissiales et dans leur rôle d'encadrement des campagnes, la notion de territoire délimité est encore peu présente. Aucun document n'évoque la constitution d'une paroisse par ajout de fragments de territoires, comme c'est le cas en Touraine ${ }^{37}$ ou même en Limousin ${ }^{38}$. La première délimitation de paroisse date, en Quercy, de 1113. Il est évidemment malaisé de dire si cette première allusion tardive à une paroisse aux contours finis traduit un phénomène déjà abouti ou s'il reflète un processus encore en cours d'élaboration. La multiplication des bornages, souvent ponctuels, aux $\mathrm{XII}^{\mathrm{e}}$ et au XIII ${ }^{\mathrm{e}}$ siècle, laisse penser que la fixation des contours paroissiaux est dans certains secteurs encore inaboutie. Il faut distinguer les zones de peuplement ancien (par exemple le pays de Vaux entre Montauban et Cahors) des zones où la densification de l'habitat parait plus récente. Dans les premiers, la territorialisation est globalement achevée avant 1250. La localisation des lieux se fait systématiquement dans le cadre paroissial. En revanche, les zones situées plus au sud, sur les terrasses du Tarn ou de la Garonne, sont traversées de conflits assez fréquents jusqu'au début du $\mathrm{XIV}^{\mathrm{e}}$ siècle ${ }^{39}$. Cette période ne marque pas pour autant une stabilisation du maillage des paroisses. La rupture entre le Moyen Âge et l'époque moderne est sur ce point complètement artificielle. On continue à assister ponctuellement à des abandons de paroisses, à des regroupements, et à des modifications de contours, et ce jusqu'à la Révolution. Si la chronologie du phénomène est à peu près connue, il est beaucoup plus malaisé d'expliquer les modalités qui ont abouti au maillage tel que nous le connaissons à la fin du Moyen Âge.

La diversité des processus de formation

18 La question du processus de formation des paroisses a fait l'objet de constructions parfois attractives intellectuellement, mais reposant sur un assemblage d'hypothèses souvent fragiles. La plus célèbre est celle du démantèlement des paroisses primitives, sorte de mitose parthénogénétique. Ce schéma est théorique et ne trouve pas, dans la documentation, de réels indices de déroulement. J'ai donc préféré m'appuyer sur des concepts moins théoriques pour tenter d'expliquer la morphogenèse de ces paroisses. Après avoir réalisé une prosopographie d'un peu plus de 200 paroisses du bas Quercy, il 
me semble possible de regrouper les facteurs d'explication en trois ensembles très inégaux: le contexte historique contemporain de la phase de mise en place du maillage ; le passé ; et le contexte géographique, impossible à ignorer. Ces ensembles se recoupent et interfèrent évidemment les uns avec les autres. Je les examinerai successivement en commençant par le passé qui semble parfois avoir joué un rôle prépondérant. En considérant que la notion de grande paroisse mérovingienne n'a aucune réalité dans la documentation, je m'appuierai surtout sur les cadres territoriaux qui transparaissent du $\mathrm{Ix}^{\mathrm{e}}$ au début du $\mathrm{Xl}^{\mathrm{e}}$ siècle pour tenter de mettre en évidence l'origine de telle ou telle paroisse.

19 L'unité fondamentale du découpage territorial à cette époque est la vicaria. Elle est très présente, quelle que soit la source : cartulaire de Saint-Théodard ou de Saint-Sernin, chartrier de Moissac. Il a été possible de reconstituer une trame sans doute incomplète du maillage des vicariae ${ }^{40}$. Plusieurs de ces entités ont pour centre un pôle ecclésial, futur chef-lieu de paroisse. C'est le cas des vicariae de Saint-Urcisse, Saint-Christophe, Saint-Jean, Flaugnac. Mais si le centre administratif se confond avec celui d'une paroisse, il n'en est rien pour le territoire. Celui des vicaria peut recouvrir plusieurs dizaines de paroisses postérieures.

Il faut donc chercher dans des cadres géographiques moins vastes. Deux répondent à cette définition, avec là encore des variantes de vocabulaire d'une zone à une autre et d'une période à une autre, l'aro et la villa. L'aro est une structure assez mal connue. Elle est mentionnée à 54 reprises en Quercy durant cette période, dans les archives des abbayes de Moissac, Saint-Théodard et Saint-Antonin-Noble-Val. Il s'agit d'une entité géographique qui sert presque exclusivement à localiser un bien. Étymologiquement, elle semble devoir être rattachée à l'ager, présent dans le Mâconnais ${ }^{41}$. Mais rien ne permet de savoir s'il s'agit d'un espace fini ou d'une nébuleuse de terroirs aux contours poreux. Il n'existe aucun texte qui fournisse des contours à un aro. En revanche, il est probable qu'une partie des paroisses de la fin du Moyen Âge découle de ces structures. Sur les 54 ari mentionnés, 32 ne sont pas identifiés, 2 correspondent à des cammas ${ }^{42}, 2$ à d'autres lieux et 18 deviennent des paroisses. Mais la continuité entre ces 18 ari et les 18 paroisses du même nom est loin d'être assurée. Ainsi, au début $\mathrm{du} \mathrm{XI}^{\mathrm{e}}$ siècle, le cartulaire de Saint-Théodard fournit la mention d'un alleu dans l'aro de Campredon ${ }^{43}$. Or Campredon ${ }^{44}$ est une paroisse qui traverse tout le Moyen Âge et l'époque moderne. Pourtant, un second texte, toujours issu du même cartulaire, signale l'existence d'un autre aro (aro de Py) situé dans la parochia de Saint-Pierre-de-Campredon ${ }^{45}$. Nous serions donc en présence d'une paroisse issue d'un aro mais dont l'étendue englobait un autre aro. Cette remarque faite pour les ari est sans doute valable pour les villae. Ces dernières sont présentes dans la documentation dès l'époque mérovingienne. Différentes vies de saints font état de dizaines de villae, pas toujours identifiées. Il faut reconnaître que l'immense majorité des villae identifiées sont devenues des centres paroissiaux. A contrario, on peut se demander si la disparition de certains toponymes n'est pas le résultat de la disparition de la villa, et donc d'une absence de continuité entre villa et paroisse. Le lien entre ces deux entités est certain, mais il ne doit pas être généralisé. Dans le secteur de Flaugnac nous disposons de mentions de plusieurs villae $\mathrm{du} \mathrm{VII}^{\mathrm{e}}$ siècle (Flaugnac, Cornus et Pern) devenues des paroisses limitrophes ${ }^{46}$. Mais, dans le même texte, apparaît une quatrième villa qui, elle, a de fortes chances de pouvoir être identifiée avec une simple cammas, également située dans le même secteur (Cougournac ${ }^{47}$ ). 
21 En outre, de même que pour les ari, rien ne permet d'affirmer que la villa correspond à un territoire précisément délimité plutôt qu'à un ensemble de terres et d'habitats discontinus. Enfin, dans l'hypothèse où une villa ou un aro a pu servir de cadre à la constitution d'un territoire paroissial, ce qui est très probable, il est difficile d'appréhender avec précision l'impact de modifications ponctuelles liées à la situation économique, sociale et politique des $\mathrm{XI}^{\mathrm{e}}-\mathrm{XII}^{\mathrm{e}}$ siècles. Il est probable que le processus de territorialisation des paroisses résulte, dans bien des cas, de la conjugaison d'entités passées et de recombinaisons présentes. Il sera sans doute impossible de mesurer précisément la part de l'un et de l'autre.

22 Je ne prendrai que deux exemples illustrant le phénomène. É. Lorans a montré, pour le Lochois, des cas de paroisses dont le territoire apparaît, au IX ${ }^{\mathrm{e}}$ siècle, comme une nébuleuse de droits (des dîmes) sans aucune continuité territoriale. La base de perception de ces droits est le manse. Aucun texte quercynois n'évoque directement ce phénomène. En revanche, comme nous l'avons déjà évoqué, il existe des mentions de décimaires qui semblent être limités à l'étendue d'un mas. De même, à la fin du Moyen Âge apparaissent plusieurs exemples de paroisses dont le territoire est discontinu et correspond en réalité à une simple addition de mas. C'est le cas de la paroisse de SaintDenis-del-Fustin, faite de trois blocs ${ }^{48}$. On peut lire dans ces découpages un mode de constitution finalement assez proche de celui que décrit la charte de Favars, sorte de charcutage sans doute issu d'âpres négociations entre patrons, recteurs et éventuellement évêque du lieu.

23 Mais si cette structure a dû jouer un rôle important, on peut aussi repérer ici et là la marque d'autres entités territoriales contemporaines. Ainsi, entre les paroisses de Flaugnac et de Cornus déjà évoquées, la limite suit précisément celle de la juridiction telle qu'elle est définie en 1292 dans la charte de coutumes de Castelnau-Montratier ${ }^{49}$. Seule une anomalie apparaît autour du mas de Laure. Ce dernier est situé à l'emplacement d'un village déserté vers 1360 , la Graulière. Il est difficile d'imaginer qu'il n'existe pas un lien entre l'anomalie de limite et l'existence de ce village dont les origines ne sont pas antérieures au XII ${ }^{\mathrm{e}}$ siècle. Si les villae du $\mathrm{VII}^{\mathrm{e}}$ siècle ont pu servir de matrice, il est certain que la définition du territoire paroissial s'est nourrie d'autres apports. L'impact de l'époque féodale et des structures de peuplement des $\mathrm{XI}^{\mathrm{e}}$-XIII ${ }^{\mathrm{e}}$ siècles sur la constitution des paroisses est sans doute plus important qu'il n'y paraît à première vue.

La complexité du phénomène relativise également la part des éléments naturels (topographie, hydrographie) dans la délimitation des paroisses. J'ai précisément analysé le contour de 222 paroisses du bas Quercy et du nord Toulousain. Ces entités étaient délimitées par un linéaire s'étirant sur $2741 \mathrm{~km}$. Au total, les cours d'eau ${ }^{50}$ servent de support de limite sur $1225 \mathrm{~km}$, soit un peu plus de $44 \%$, ce qui laisserait croire à un très fort déterminisme géographique. Là encore, la réalité est toute en nuance. S'il est certain que les cours d'eau ont formé des contours faciles à identifier et relativement stables, ils n'ont pas toujours constitué des limites fortes. Ainsi la vallée de l'Aveyron, mais aussi celle du Lot, abritent des paroisses qui s'étendent sur les deux rives ${ }^{51}$. En outre les chemins constituent également un support très important pour les contours paroissiaux ( $37 \%$ du linéaire), ce qui relativise le rôle des rivières. On pourrait faire la même remarque avec les crêtes des serres qui dominent la vallée du Tarn.

Si l'existence d'un maillage sans doute déjà dense d'églises rurales à l'époque carolingienne ne fait aucun doute, la formation des paroisses semble finalement assez 
tardive. L'apparition du mot dans les actes de la pratique est globalement rare avant la fin du $x^{e}$ siècle. En Quercy, il faut même attendre la seconde moitié du siècle suivant pour voir l'abandon du système de localisation par la vicaria au profit de la parochia. Même s'il est sans doute risqué de généraliser à partir d'un corpus documentaire se limitant à quelques centaines de textes, il faut constater que les éléments de définition de la paroisse territoriale n'existent pas avant 1113, date de la première délimitation de paroisse quercynoise. Le phénomène de la territorialisation de la paroisse est donc tardif et sans doute polymorphe. Il faut éviter d'expliquer cette territorialisation par un déterminisme trop accentué. Si une partie de ces territoires paroissiaux tire son origine de situations passées, les remodelages ponctuels sont sans doute assez fréquents. Cela s'accorde d'ailleurs assez bien avec l'importance des conflits sur ces délimitations jusqu'à la fin du XIII ${ }^{\mathrm{e}}$ siècle, en particulier dans les zones de peuplement récent ou de remodelage du peuplement ancien (bastides). Enfin, même si la fin du XIII e siècle semble marquer un optimum dans la structuration du maillage paroissial, il faut reconnaître que ce dernier est resté vivant et donc instable durant tout le Moyen Âge et l'époque moderne. L'abandon et la ruine d'églises paroissiales ne sont pas un apanage du Moyen Âge, pas plus que la mise en place de nouvelles entités ou le déplacement ponctuel de certains édifices.

\section{NOTES}

1. P. Imbart de la Tour, Les Origines religieuses de la France. Les paroisses rurales $d u I^{e}$ au XI siècle, Paris, 1900 (rééd. 1979).

2. F. Hautefeuille, Structures de l'habitat rural et territoires paroissiaux en bas-Quercy et haut Toulousain $d u$ VII $a u$ XIV $v^{e}$ siècle, Thèse de doctorat nouveau régime, université Toulouse II-le Mirail, 1998.

3. Pour se limiter à l'essentiel : Alle origini della parrocchia rurale (IV-VIII sec.). Actes du Colloque de Rome, 19 mars 1998, Ph. Pergola, P.M. Barbini éd., Vatican, 1999 ; Ch. Delaplace, «Les origines des églises rurales ( $\mathrm{v}^{\mathrm{e}}$-vi $\mathrm{vi}^{\mathrm{e}}$ siècles). À propos d'une formule de Grégoire de Tours ", Histoire et Sociétés Rurales, 18, 2002, p. 11-40 ; Aux origines de la paroisse rurale en Gaule méridionale, IV ${ }^{e}-I X^{e}$ siècles. Actes du Colloque de Toulouse, 21-23 mars 2003, C. Delaplace éd., Toulouse, 2005.

4. L. d'Alauzier, « La Tulmonenc jusque vers 1350 », Bulletin de la Société Archéologique du Tarn-et-Garonne, 88, 1961, p. 77-87.

5. $\mathrm{AD} 82, \mathrm{G} 239$ et $\mathrm{G} 240$.

6. Les archives de Moissac ont été éditées, mais non publiées (M.-C. Lefebre, Les plus anciennes chartes de l'abbaye de Moissac, Thèse de l'École nationale des chartes, 5 vols, Paris, 1967).

7. La reconstitution des contours paroissiaux s'appuie sur les textes de la fin du Moyen Âge ou, lorsque ceux-ci ne sont pas suffisants, sur les sources fiscales modernes, tels les compoix.

8. Voir ici-même les contributions de M. Lauwers et É. Zadora-Rio. 
9. Je pense en particulier à celles du concile de Latran IV et à la définition d'Hostiensis (Hostensis, Summa aurea, III [De parochiis]).

10. Ch.-Éd. Perrin, J. de Font Réaulx, Pouillés des provinces d'Auch, de Narbonne et de Toulouse, Paris, 1972.

11. Par exemple, la parrochialis ecclesia Sancti Agapyti de Tauriaco, dans l'archiprêtré de Gignac, ibid., p. 446.

12. Ibid., p. 447 : parrochia Sancti Saturnini de Valeyra et parrochia Sancti Palary.

13. Ibid., p. 461.

14. Canton de Lalbenque, commune de Montdoumerc (46).

15. Ms. BnF, lat. 17674, f. 150.

16. La première mention du castrum date de 1259 : AN JJ 11, f. $86 \mathrm{~V}^{\circ}$.

17. Ch.-Éd. Perrin, J. de Font Réaulx, op. cit., p. 443 : «Ecclesia de Calviaco, capellania valet nihil quia prior rapit totum.»

18. C'est le cas des communes de Lauzerte (82) et de Castelnau-Montratier (46).

19. Éd. Albe, "Autour de Jean XXII : Jean XXII et les familles du Quercy ", Annales de Saint-Louis-des-Français, 7, 1902, p. 460, 468, 486.

20. C'est le cas du prieuré de Bioule sur les rives de l'Aveyron.

21. Dans ce cas de figure, les vocables sont distincts. C'est par exemple le cas de Bioule 82 sur la rive de l'Aveyron (Saint-Sauveur et Saint-Pierre) ou à Bressols sur les rives du Tarn (Saint-Martin et Saint-Benoît). À Bioule, c'est le vocable du prieuré cure, SaintSauveur, qui s'est imposé (l'église paroissiale primitive a disparu dès la fin du Moyen Âge). À Bressols, l'église paroissiale qui traverse le Moyen Âge prend un double vocable, Saint-Benoît et Saint-Martin.

22. Ainsi à Bressols au sud de Montauban, la documentation fait apparaître trois édifices de part et d'autre de la rivière. Deux d'entre eux ont eu le statut de prieuré. Une seule de ces églises a survécu jusqu'à nos jours.

23. Commune du canton de Lalbenque (46).

24. Ainsi à Castelnau-Montratier (46), suivant les phases suivantes : d'abord la constitution d'un espace paroissial intra muros (xiiie-xive siècles), puis l'abandon de l'église champêtre dont le territoire paroissial est rattaché à l'église castrale (fin $\mathrm{xv}^{\mathrm{e}}$ siècle).

25. Castrum de Pechpeyroux, Montpezat, Sauveterre..., cf. F. Hautefeuille, op. cit., II, p. 91.

26. N. de Peña, Documents sur la maison de Durfort (Xle-XVe siècles), Bordeaux, 1977, I, $\mathrm{n}^{\circ} 270$.

27. M. Aubrun, La Paroisse en France des origines au XVe siècle, Paris, 1986.

28. J. Avril, «La "paroisse" dans la France de l'an Mil », dans M. Parisse, X. Barrali Altet éd., Le Roi de France et son royaume autour de l'an mil. Actes du Colloque Hugues Capet, Paris-Senlis, 22-25 juin 1987, Paris, 1992, p. 203-218.

29. BnF, Fonds Doat, vol. 89, f. 117.

30. J. Lartigaut, «La paroisse rurale en Quercy au $\mathrm{xv}^{\mathrm{e}}$ siècle », dans Montauban et le basQuercy, Actes du XXVII ${ }^{e}$ congrès d'études de la Fédération des sociétés académiques et savantes de Languedoc-Pyrénées, Montauban, 1974, p. 213 et F. Hautefeuille, op. cit., II, p. $450-452$.

31. Chef-lieu de canton de l'arrondissement de Montauban (82).

32. La première mention de cette paroisse est moderne.

33. C. Baraut, Les Actes de consagracions d'esglésies de l'antic bisbat d'Urgell (segles IX-XII), Urgell, 1986 ; voir aussi J.-P. Illy, Les Églises et paroisses rurales de l'évêché d'Urgell, du début 
du IX siècle aux alentours de 1040, Mémoire de maîtrise dactyl., université Toulouse II-Le Mirail, 1988.

34. P. Ourliac, Cartulaire de l'abbaye de Lézat, Paris, $1984, \mathrm{n}^{\circ} 477$, parrochia Sancti Martini de Nuer, attestée entre 989 et 994.

35. P. Ourliac, A.-M. Magnou, « Les paroisses de l'abbaye de Lézat », Cahiers de Fanjeaux, 19, 1984, p. 346-347.

36. F. Hautefeuille, « La cartographie de la paroisse et ses difficultés de réalisation », Aux origines de la paroisse rurale, op. cit., p. 24-32.

37. É. Lorans, Le Lochois du haut Moyen Âge au XIII ${ }^{e}$ siècle. Territoires, habitats et paysages, Tours, 1996, p. 73-75.

38. Je remercie D. Iogna-Prat d'avoir souligné la prudence qui doit être de mise lorsque l'on évoque la célèbre charte de fondation (897) de la paroisse de Favars en Limousin (cf. M. Aubrun, L'Ancien Diocèse de Limoges des origines au milieu du XI siècle, ClermontFerrand, 1981, p. 351). La mention d'un sceau épiscopal laisse planer un très sérieux doute sur l'acte qui semble au minimum interpolé.

39. F. Hautefeuille, op. cit., II, p. 499.

40. Cf. ma contribution à l'Histoire du Tarn-et-Garonne, J.-C. Fau, Saint-Jean-d'Angély, 2003, p. 77.

41. F. Bangé, «L'ager et la villa, structures du paysage à la fin du haut Moyen Âge », Annales ESC, mai-juin 1984, p. 529-569.

42. En Quercy, le cammas (caput mansi) correspond soit à un mas plus peuplé que les autres, soit à un mas doté d'un statut fiscal particulier, vers lequel converge une partie des redevances foncières des environs.

43. AD 82, G 239, f. 39.

44. Commune de Montastruc (82).

45. AD 82, G 239, f. 73.

46. Vita Desiderii Cadurcae urbis episcopi, MGH, Scriptores, 4, p. 587.

47. Cougournac est un hameau de la commune de Castelnau-Montratier (46). Mais un doute subsiste car il existe une paroisse du même nom à une trentaine de kilomètres plus au sud, commune de Puycornet (82).

48. Communes de Vazerac et Cazes-Mondenard (82), cf. F. Hautefeuille, op. cit., II, p. 495.

49. L. Limayrac, Étude sur le Moyen Âge, histoire d'une communauté et d'une baronnie du Quercy (Castelnau-Montratier), Cahors, 1885 (la charte est publiée en annexe).

50. Cela inclut aussi bien des rivières majeures comme le Tarn que des rus intermittents.

51. C'est le cas de la paroisse de Cos, commune de Lamothe-Capdeville (82) sur l'Aveyron et de Notre-Dame-de-Velle, commune de Vers (46) sur le Lot.

\section{RÉSUMÉS}

Dans l'aire de la confluence Tarn-Aveyron-Garonne, la documentation fait apparaitre plusieurs types de paroisses, attachées à différents types d'églises, de l'église annexe au prieuré. Ces 
différences paraissent plus juridiques ou fiscales que réelles dans le fonctionnement quotidien de la paroisse des paroissiens. Par ailleurs, le processus de territorialisation des paroisses semble tardif et lent à se généraliser. Il s'explique par trois phénomènes qui se mêlent : l'influence de structures issues du passé, la contrainte géographique et les aléas de l'histoire contemporaine du processus de formation.

The process of bounding parish territories in Quercy (10th-15th c.). In the confluence area of the Tarn, the Aveyron and the Garonne, documentary evidence shows a wide variety of parishes, attached to different kinds of churches (secondary church, monastic church...). These differences seem to bear more on legal or fiscal status than on the reality of the parish or on parishioners'practice. The process of bounding parish territories was late, and its completion was slow to come about. It was determined by three inter-related factors: the influence of patterns of the past, the topographical constraints, and incidental local circumstances.

INDEX

Keywords : territory, priory, hierarchy, tithes

Mots-clés : territoire, Quercy, prieuré, hiérarchie, dîmes

\section{AUTEUR}

\section{FLORENT HAUTEFEUILLE}

Université de Toulouse II-Le Mirail, UMR 5136-FRAMESPA, Maison de la recherche, 5, allée Antonio Machado, F-31058 Toulouse cedex 01 